\title{
COMPLICATIONS OF PERCUTANEOUS NEPHROLITHOTOMY IN PRIVATE PRACTICE
}

\author{
Ottra Ramesh ${ }^{1}$, Kilambi Satyanarayana Chary², Muthyalapati Gopichand ${ }^{3}$, Ravi Prabhu Gottumukkala 4 \\ ${ }^{1}$ Consultant, Department of Urology, Sneha Hospital, Tirupati. \\ ${ }^{2}$ Consultant, Department of Urology, Sneha Hospital, Tirupati. \\ ${ }^{3}$ Consultant, Department of Urology, Sneha Hospital, Tirupati. \\ ${ }^{4}$ Professor \& HOD, Department of Community Medicine, ACSR Govt. Medical College, Nellore.
}

\section{ABSTRACT}

\section{BACKGROUND}

The increasing global prevalence of nephrolithiasis continues to burden the health care delivery systems of developing nations. Percutaneous Nephrolithotomy (PCNL) is considered the standard treatment for many types of calculi. This study focuses on the complications of PCNL in private practice setting at a peripheral center using the modified Clavien system and role of Guy's stone score as a predictor of stone free rate and complications.

\section{METHODS}

This is a prospective cohort study of 480 patients who underwent PCNL during August 2011 to July 2015. The complications were classified according to modified Clavien system and correlated with the stone complexity as per the Guy's stone score.

\section{RESULTS}

It was found that overall 120 complications were reported in 480 patients with the incidence of complications of Grade I, II, IIIa, IIIb, IVb being 48 (10\%), 38 (7.9\%), 15 (3.5\%), 12 (2.5\%) and 4 (0.8\%) respectively. As per the Guy's stone score there were 336, 104 and 40 cases belonging to GSS I, II and III respectively. All grades of complications were more common in GSS II and III. The stone clearance was found to be complete in $95 \%, 82 \%$ and $75 \%$ of GSS I, II, III respectively.

\section{CONCLUSION}

The stone complexity is related to complication rate and GSS helps to predict stone free rate and complications.

\section{KEYWORDS}

Nephrolithiasis, Percutaneous Nephrolithotomy, Guy Stone Score, Clavien System, Renal Calculi.

HOW TO CITE THIS ARTICLE: Ramesh 0, Chary KS, Gopichand M, et al. Complications of percutaneous nephrolithotomy in private practice. J. Evolution Med. Dent. Sci. 2016;5(22):1175-1179, DOI: 10.14260/jemds/2016/273

\section{INTRODUCTION}

The increasing global prevalence of nephrolithiasis continues to burden the health care delivery systems of developing nations and extracts a disproportionate toll on populations of the developing world. In India alone, the prevalence of nephrolithiasis is nearly twice the rate reported in 1990. Percutaneous entry into the collecting system was first described in 1950s, but it was not until the mid 1970s and 1980s that percutaneous access to the renal collecting system was utilized routinely for the removal of nephrolithiasis. Although Percutaneous Nephrolithotomy (PCNL) initially proved to be an effective technique, the concurrent introduction of Shock Wave Lithotripsy (SWL) resulted in a rapid and marked decrease in the utilization of PCNL. There has been however a recent increase in the utilization of PCNL, largely attributable to the limitations of newer SWL equipment.

Greater surgeon experience and improvements in instruments (Mini PCNL, Ultramini PCNL and Micro PCNL) and

Financial or Other, Competing Interest: None.

Submission 28-01-2016, Peer Review 27-02-2016,

Acceptance 04-03-2016, Published 16-03-2016.

Corresponding Author:

Dr. Ravi Prabhu Gottumukkala,

Professor \& HOD,

Community Medicine,

ACSR Govt. Medical College,

Nellore.

E-mail: raviprabhu1610@gmail.com

DOI: $10.14260 /$ jemds $/ 2016 / 273$ lithotripsy technology (Holmium YAG laser) have increased the efficiency of PCNL.1

PCNL is considered the standard treatment for staghorn calculi and large volume renal calculi as well as upper tract calculi refractory to other modalities, difficult lower pole stones, cysteine stones and calculi in anatomically abnormal kidneys. PCNL is well tolerated procedure, but as with any surgical intervention is associated with a specific set of complications.

An international multinational study of 5,803 patients undergoing PCNL reported an overall complication rate of $21.5 \%$. The study conducted by the clinical research of the Endourological Society (CROES).(1) utilized the modified Clavien system for reporting complications.(1) The modified Clavien system was validated in general surgery and is being adopted by urologists for grading of complications in urology. 2,3

The rate of complications varies according to the complexity of stone disease. The stones are generally classified into single/multiple, pelvic/calyceal and simple/staghorn which may be a partial /complete staghorn. Guy's stone score (GSS) was conceived and validated for better prediction of the complications and stone free rates after PCNL. 4

The private practice has its own constraints, especially in a peripheral centre. We report our perioperative complications of PCNL according to modified Clavien system, in which the stone complexity has been classified using Guy's stone score. 


\section{MATERIALS AND METHODS}

This is a prospective observational study on 480 patients undergoing percutaneous nephrolithotomy. All patients who were operated between June 2011 and August 2015 with percutaneous nephrolithotomy were included in the study. That means those cases with certain comorbidities like diabetes mellitus, hypertension and chronic obstructive pulmonary obstructive disease were also included in the study. Permission was obtained from the Institutional Ethical Committee of Sri Venkateswara Medical College, Tirupati. The exclusion criteria included those cases with complete staghorn calculi and those with proven recurrence of calculi. A written consent was obtained from all patients who were recruited for the research study. The patients were explained clearly the purpose of the study in local language by the investigators. Preoperative surgical workup included route investigations like complete blood picture, sugar levels, urine routine, urine culture, serum creatinine, HIV, HBV and HCV testing. Radiographic evaluation included X-ray of Kidney Ureter Bladder (KUB), Ultrasound, IVU or NCCT. Complexity of stone analysis was done based on Guy's stone score and was given in Table 1.

Patients underwent PCNL under spinal anaesthesia in almost all cases except children $<6$ years of age and cases with upper calyceal puncture in high lying kidney when general anaesthesia was used. Antibiotic prophylaxis was given to all patients.

Patients underwent cystoscopy and ureteric catheterization in lithotomy position. Unimpacted stones in upper ureter were pushed into the kidney with urethroscope and impacted stones were partially fragmented and pushed into the kidney and were removed by PCNL.

The lower calyceal puncture was preferred for lower calyceal and pelvic calculi. Middle calyceal puncture was done for middle calyceal stone or pelvic stone where the lower calyceal axis was not convenient to reach the pelvis. Upper calyceal puncture was done for upper calyceal stone. A Terumo guidewire was positioned in the ureter whenever possible. The track dilatation was done with coaxial metal Alken dilators up to $22 \mathrm{~F}$. When the guidewire did not go to the pelvis because of stone impaction in the calyx, dilatation was done upto $20 \mathrm{~F}$ only and $15.5 \mathrm{~F}$ nephroscope was used to enter the pelvi-calyceal system under vision. Stones measuring $1 \mathrm{~cm}$ were removed intact and larger ones are fragmented with pneumatic lithotripter. The stone clearance was confirmed by intraoperative fluoroscopy.

A Double J stent was passed antegradely when there was injury to the pelvis/PUJ/ureter. Nephrostomy tube was clamped for 6-12 hours if there was significant bleeding intraoperative or the return was bloody at the end of procedure.

The patient was evaluated after 48 hours by plain X-ray KUB and Ultrasonography. If the stone clearance was complete, nephrostomy tube is removed. If there were residual fragments which needed a relook nephrostomy, the nephrostomy tube was retained and relook was done after 4-5 days.

The PCNL procedure was considered successful if the patient was stone free or the stone fragments were less than 4 $\mathrm{mm}$ (Clinically insignificant residual stone fragments). The data included age, sex, GSS stone score, number of punctures, punctured calyx, site of puncture in relation to the last rib, number of operative punctures and mean duration of surgery. The modified Clavien grading system (Table 2) was used for evaluating peri- and post-operative complications. ${ }^{5}$

\section{RESULTS}

The data from all the 480 patients who underwent PCNL during the study period from 2011 to 2015 were analysed using Epi Info 7.1.4 version available freely from CDC (Center for Disease Control) of Atlanta, USA. The categorical variables were expressed as proportions, while continuous measures were expressed as mean with standard deviation. The differences between proportions were analysed using Chisquare test. The GSS (GUYS stone score) was used to classify the stones. The descriptive details of the cohort were summarized in Table 3. Majority of the calculi belonged to GSS I (70.0\%) followed by GSS II (21.7\%) and GSS III (8.3\%). The following are the sub-types within each category.

GSS1 (336): Primary pelvic (156), PUJ (90), upper ureteric stones pushed into renal pelvis (50), lower calyceal (40) and middle calyceal stones (10).

GSS2: Multiple calculi (70), upper calyceal calculi (20) and calculi in abnormal anatomy (14).

GSS3: Partial staghorn calculi (40).

The stone clearance was complete in one session in $90 \%$ of the patients and 2 nd session was required in $2 \%$ of patients. The stone clearance was incomplete in $8 \%$ of the patients.

One hundred and twenty complications were seen in 480 patients as shown in Table 4. The occurrence of complications was correlated with Guy's stone score. The commonest complication, fever was seen in $10 \%$ of total patients and as per the GSS score it was seen in $6.8 \%, 14.4 \%$ and $25 \%$ of GSS I, II and III respectively.

Blood transfusion was required in $7.9 \%$ of total cases and as per the GSS score it was 3.3\%, $11.5 \%$ and $37.5 \%$ in GSS I, II and III respectively. Preoperative cross matching was done for all GSS III cases and for the other groups cross match was done only when blood transfusion was required.

Intraoperative bleeding resulting in poor vision and incomplete clearance of the fragments requiring relook and clearance after 1 week occurred in 10 patients, out of which 6 belonged to GSS III and IV to GSS II. Four patients required clot evacuation in the postoperative period. Two of them belonged to GSS I and the other 2 belonged to GSS II. The 2 patients in GSS I who required clot evacuation also needed angioembolization because of recurrent haematuria.

Mucosal pelvicalyceal injury occurred in 15 patients in PUJ and pelvicalyceal system. They were managed by DJ stenting in 6, 5 and 4 patients who belonged to GSS I, II and III categories respectively. Urosepsis requiring ICU care occurred in 1, 1 and 2 patients belonging to GSS I, II and III categories respectively.

Stone clearance was achieved in $97 \%$ of GSS I, $82 \%$ of GSS II and $75 \%$ of GSS III patients. A second session was required to clear the residual stones, which was due to the poor vision on account of bleeding. It occurred in GSS II and III categories.

\section{DISCUSSION}

PCNL is recommended as the standard treatment for renal calculi $>2.5 \mathrm{~cm}$, partial and complete staghorn calculi. ESWL is preferred for small size calculi. RIRS is emerging as the treatment of choice for smaller calculi. ${ }^{6}$ 
In private practice the situation is not ideal, especially for patients with low socio-economic status and in government sponsored health insurance where the package is nonnegotiable and complete stone clearance is mandatory to get that package. RIRS is not economically viable for these patients and ESWL with unpredictable success rate for lower pole stone, cystic stone and the need for ancillary procedure like ureteroscopy is not an ideal option. This makes the PCNL as the preferred choice for upper ureteric stone $>1 \mathrm{~cm}$, pelvic stone $>1.5 \mathrm{~cm}$ and lower calyceal stone $>1 \mathrm{~cm}$ to achieve complete clearance without the need for ancillary procedures.

The complication rate of PCNL may vary widely from $20 \%$ to $83 \%$ and hence the true complication rate of PCNL is difficult to compare. ${ }^{7}$ The means by which the data are obtained and reported probably have an impact on the complications rate as the procedure. Modified Clavien grading system has been shown to be a reliable tool for more objective comparison of outcome in urology.8,9,10,11,12 Complications graded as Clavien Grade 1 and 2 are considered as minor, while Grades 3, 4 and 5 are considered as major. Our overall complication rate among 480 consecutive patients of PCNL is $25 \%$. Among them 70\% belonged to Clavien Grade 1, 2 (Minor) and 30\% belonged to Clavien Grade 3, 4 (Major).

Fever in the postoperative period was the most common complication (14\%). The reported incidence of fever after PCNL varied from as low as $2.8 \%$ (5) to $27-30 \%$. It may be because of the different patient population and the policy regarding the use of antibiotics. It is our policy to give the patients culture specific antibiotics when urine culture was positive preoperatively and to give Inj. Cefoperazone $(1000 \mathrm{mg}$ + Sulbactam $500 \mathrm{mg}$ ) for others which was continued for 48 hours postoperatively. The patients who developed intraoperative/postoperative rigors are given additional coverage with Inj. Amikacin (500 mg) Factors predisposing to fever after PCNL include pre-existing UTI, infected stone, diabetes mellitus, renal insufficiency, the amount pressure of the irrigation fluid and the duration of surgery. ${ }^{13,14}$

Bleeding requiring blood transfusion was the second most common complication observed in $7.9 \%$ patients. This is comparable to the $5.7 \%$ reported in the CROES (Clinical Research Office of the Endourology Society) group. However, an overall transfusion rate of $24 \%$ has been reported by Mandal et al.15 The procedure was abandoned due to intraoperative bleeding and repeats PCNL was done in 10 patients. Two patients required $(0.04 \%)$ super selective angioembolization due to recurrent, severe haematuria with clot retention in the postoperative period. The rate of intervention for renal haemorrhage has been reported to be $0.6-1.4 \%$ in the review by Skolarikos and Rosette. ${ }^{13}$

Bleeding is the most feared complication of PCNL. Puncture is always attempted through the posterior calyx aiming the cup of the calyx avoiding the hypervascular region adjacent to the infundibulum. The nephrostomy tube is kept clamped at the end of the procedure in case of significant bleeding to provide intrarenal tamponade. The increased stone complexity is directly associated with the need for transfusion. ${ }^{15}$ Multiple attempts at initial puncture increase the risk of bleeding. Additional risk factor includes torque of the instrument, infection, renal insufficiency and prolonged operation time. ${ }^{16}$

Renal collective system injury occurred in 3\% patients, in our series majority of them were mucosal injuries without any clinical significance. Injury to the collecting system can occur during dilatation of the tract, during fragmentation of hard stone and due to inadvertent injury to the mucosa with forceps during extraction of stone fragments.

Urosepsis requiring ICU management with Oxygen inhalation, vasopressors and broad-spectrum antibiotics was seen in $(0.8 \%)$ patients. Female gender, multiple punctures, struvite calculi and positive pelvic urine and stone cultures are risk factors for the development of Systemic Inflammatory Response Syndrome.14

Plural injury is seen in $3-7 \%$ of supracostal punctures. ${ }^{17}$ Though we have done intercostal puncture in nearly 30\% patients, we have not encountered any pleural injury. Because the pleura and diaphragm are related to the upper pole of the kidney, upper pole punctures are commonly associated with plural injury. Though we have done upper pole puncture in 20 patients, plural injury was not encountered, probably because we punctured through $11^{\text {th }}$ intercostal space in all the intercostal punctures and not through $10^{\text {th }}$ intercostal space.

The colonic injury was reported as $0.2-0.8 \%$ of patients in the review by Skolaris and Rosette. ${ }^{13}$ We have not encountered any colonic injury in our patients. The risk factors for colon injury are retrorenal colon, thin patient and previous open renal surgery. We have not encountered any colonic injury, though some of our patients had the above risk factors.

The GSS accurately predicted the stone free rate after PCNL in our study, as it was by Mandal et al. ${ }^{15}$ It was easy to use, reproducible, objective and reliable method for describing the complexity of the stone and predicts SFR after PCNL. The stone clearance was $97 \%$ in GSS1 and $82 \%$ in GSS2 and $75 \%$ in GSS3.

\section{CONCLUSION}

This is a prospective study of complications of PCNL in a private practice setting at a district centre, where there are several constraints like complete stone clearance in one sitting is necessary, especially for patients with government sponsored health schemes where there is no scope for upward revision of the package, inadequate availability of blood and lack of angio-embolization facility. The proper selection of patient is very important in private practice in a peripheral centre unlike Institutional setting. The GSS helps in the selection of patients, to counsel them about stone clearance, need for blood transfusion and to avoid difficult and complicated cases. Needless to say that the expertise and the experience of the surgeon are also important to avoid major mishaps in PCNL in a private practice sitting. The social implications from patients' perspective is that those patients with pre-existing morbidities and large and recurrent calculi need to undergo at a bigger centre with better infrastructure and facilities including angio-embolization. 


\begin{tabular}{|c|c|c|}
\hline Sl. No. & Parameter & Value (\%) \\
\hline 1. & Mean age & 40.2 years (Range $2-65$ ) \\
\hline 2. & Male/Female ratio & $5: 1$ \\
\hline 3. & Classification of calculi & \\
\hline (a) & GSS I & $336(70.0)$ \\
\hline (b) & GSS II & $104(21.7)$ \\
\hline (c) & GSS III & $40(8.3)$ \\
\hline (d) & GSS IV & $0(0.0)$ \\
\hline 4. & Clearance after sessions & \\
\hline (a) & Clearance after 1 session & $432(90.0)$ \\
\hline (b) & Clearance after 2 sessions & $10(2.1)$ \\
\hline (c) & Incomplete clearance & $38(7.9)$ \\
\hline 5. & Site of puncture & \\
\hline (a) & Sub-costal & $336(70.0)$ \\
\hline (b) & Supra-costal & $144(30.0)$ \\
\hline 6. & Mean duration of operation (min) & $40 \min (30-180)$ \\
\hline
\end{tabular}

\begin{tabular}{|c|c|c|c|}
\hline Grade & Complication & No. of Patients & Percentage \\
\hline Grade I & Fever & 48 & 14.3 \\
\hline Grade II & Blood transfusion & 37 & 36.5 \\
\hline \multirow[t]{2}{*}{${ }^{*}$ Grade III a } & $\begin{array}{l}\text { Double J stent placement for ureteropelvic junction } \\
\text { and pelvic injury }\end{array}$ & 15 & 3.0 \\
\hline & Retention and colic due to blood clots & 4 & 0.8 \\
\hline \multirow[b]{2}{*}{${ }^{*}$ Grade III b } & Arteriovenous fistula & 2 & 0.4 \\
\hline & $\begin{array}{l}\text { Intraoperative bleeding requiring } \\
\text { termination of operation }\end{array}$ & 10 & 4.8 \\
\hline \multirow{2}{*}{${ }^{*}$ Grade IV a } & Neighbouring organ injury & 0 & 0.0 \\
\hline & Myocardial infarction & 0 & 0.0 \\
\hline${ }^{*}$ Grade IV b & Urosepsis & 4 & 0.8 \\
\hline
\end{tabular}

\begin{tabular}{|c|c|c|c|c|c|}
\hline Grade & $\begin{array}{c}\text { Total } \\
\mathbf{( 4 8 0 )}\end{array}$ & $\begin{array}{c}\text { GSS I } \\
(\mathbf{3 3 6})\end{array}$ & $\begin{array}{c}\text { GSS II } \\
\mathbf{( 1 0 4 )}\end{array}$ & $\begin{array}{c}\text { GSS III } \\
\mathbf{( 4 0 )}\end{array}$ & P Value \\
\hline Grade 1 & $48(10.0 \%)$ & $23(6.8)$ & $15(14.4 \%)$ & $10(25.0)$ & $<0.001 ; \mathrm{S}$ \\
\hline Grade 2 & $37(7.9)$ & $10(3.3)$ & $12(11.5)$ & $15(37.5)$ & $<0.001 ; \mathrm{S}$ \\
\hline Grade 3 a & $19(4.0)$ & $10(3.0)$ & $4(1.2)$ & $5(12.5)$ & $0.01 ; \mathrm{S}$ \\
\hline Grade 3 b & $12(2.7)$ & $2(1.8)$ & $4(2.9)$ & $6(10.0)$ & $0.01 ; \mathrm{S}$ \\
\hline Grade 4 a & $0(0.0)$ & $0(0.0)$ & $0(0.0)$ & $0(0.0)$ & $0.008 ; \mathrm{S}$ \\
\hline Grade 4 b & $4(0.8)$ & $1(0.3)$ & $1(1.0)$ & $2(5.0)$ & --- \\
\hline Grade 5 & $0(0.0)$ & $0(0.0)$ & $0(0.0)$ & $0(0.0)$ & \\
\hline \multicolumn{7}{r}{ Table 3: Comparison of Complications Classified according to the Modified } \\
\\
Clavien System between and Calculi Classified according to GSS
\end{tabular}

\begin{tabular}{|c|c|c|c|}
\hline & Total Cases & $\begin{array}{c}\text { Complete Clearance } \\
(\%)\end{array}$ & $\begin{array}{c}\text { Incomplete Clearance } \\
(\%)\end{array}$ \\
\hline GSS I & 336 & $326(97.0)$ & $10(3.0)$ \\
\hline GSS II & 104 & $86(82.7)$ & $18(17.3)$ \\
\hline GSS III & $40 \quad 30(75.0)$ & $10(25.0)$ \\
\hline \multicolumn{2}{|c|}{ Table 4: Stone Clearance Rate in Relation to GSS Rate } \\
\hline
\end{tabular}

\section{REFERENCES}

1. Rosette J, Assimos D, Desai M, et al. The clinical research office of the endourological society percutaneousne phrolithotomy global study: indications, complications, and outcomes in 5803 patients. J Endourol 2011;25:117.

2. Labate G, Modi P, Timoney A, et al. The percutaneous nephrolithotomy-global study: classification of complications. J Endourol 2011;25:1275-80.
3. Morgan M, Smith NS, Thomas K, et al. Is clavien the new standard for reporting urological complications? BJU Int 2009;104:434-6.

4. Thomas K, Smith NC, Hegarty N, et al. The guy's stone score-grading the complexity of percutaneous nephrolithotomy procedures. Urology 2011;77:277-81. 
5. Tefekli A, Karadag MA, Tepeler K, et al. Classification of percutaneous nephrolithotomy complications using the modified clavien grading system: looking for a standard. Eur Urol 2008;53:184-90.

6. Turk C, Knoll T, Petrik A, et al. Guidelines on urolithiasis. Eur Assoc Urol 2010;1-106.

7. Michel MS, Trojan L, Rassweiler JJ. Complications in percutaneous nephrolithotomy. Eur Urol 2007;51:899906.

8. Rabbani F, HerranYunis L, Pinochet $\mathrm{R}$, et al. Comprehensive standardized report of complications of retropubic and laparoscopic radical prostatectomy. Eur Urol 2010;57:371-86.

9. Ramasamy R, Afaneh C, Katz M, et al. Comparison of complications of laparoscopic versus laparo-endoscopic single site donor nephrectomy using the modified clavien grading system. J Urol 2011;186:1386-90.

10. Szydelko T, Kasprzak J, Apoznanski W, et al. Clavien classification of complications after 150 laparoscopic pyeloplasties. Urology 2011;77:1359-64.

11. Ramasamy R, Reifsnyder J, Shin B, et al. Laparoscopic and open partial nephrectomy: comparison of complications using clavien grading system. J Urol 2011;85:e610.
12. Mamoulakis C, Efthimiou I, Kazoulis S, et al. The modified clavien classification system: a standardized platform for reporting complications in transurethral resection of the prostate. World J Urol 2011;29:205-10.

13. Skolarikos A, De La Rosette J. Prevention and treatment of complications following percutaneous nephrolithotomy. Curr Opin Urol 2008;18:229-34.

14. Dogan HS, Sahin A, Cetinkaya Y, et al. Antibiotic prophylaxis in percutaneous nephrolithotomy: prospective study in 81 patients. J Endourol 2002;16:649-53

15. Mandal S, Goel A, Katpalia R, et al. Prospective evaluation of complications using the modified clavien grading system, and of success rates of percutaneous nephrolithotomy using guy's stone score: a single-center experience. Indian J of Urology 2012;28:392-8.

16. Srivastava A, Singh KJ, Suri A, et al. Vascular complications after percutaneous nephrolithotomy: are there any predictive factors? Urology 2005;66:38-40.

17. Raza A, Moussa S, Smith G, et al. Upper pole puncture in percutaneous nephrolithotomy: a retrospective review of treatment safety and efficacy. BJU Int 2008;101:599602 . 